\title{
Organizational attributes that support reliable Emergency Medical Services response during Mass Casualty Incidents in Nairobi County, Kenya
}

\author{
Evelyn Baru*, Prof. Stanley Omuterema**, Dr Janet Nabiswa*** \\ *Department of Emergency Management Studies, Masinde Muliro University of Science \& Technology Kenya \\ **Department of Disaster Management and Sustainable Development, Masinde Muliro University of Science \& Technology, Kenya \\ ***Department of Educational Psychology, Kibabii University, Kenya
}

DOI: 10.29322/IJSRP.11.11.2021.p11963

http://dx.doi.org/10.29322/IJSRP.11.11.2021.p11963

\begin{abstract}
When disaster strikes resulting in mass casualties, the number of victims rapidly exceeds locally available intervention resources thereby necessitating exceptional response arrangements. When these tragic and traumatizing large-scale events occur, the public expects the local disaster response entities to be on hand to provide much needed assistance. Whereas the ultimate goal of any emergency medical response is to obtain the best possible intervention for the greatest number of casualties, research indicates that the efficacy of the emergency medical services (EMS) organization's response to past major incidents in Nairobi County, Kenya, has been inefficient. The aim of this study was to address this gap by examining the organizational attributes of EMS organizations that influence reliable disaster response to Mass Casualty Incidents (MCIs) in Nairobi County Kenya. The specific objective of the study was to examine EMS personnel's perceptions of organizational strategies that support reliable disaster response during MCIs in Nairobi County, Kenya. The study was guided by the high reliability theory as an approach for enhancing reliable emergency medical response performance in dynamic high-risk settings such as mass casualty disaster events. Non-probability convenience sampling approach was used to draw a target study population of 71 , out of which a sample size of 54 study respondents comprising certified emergency medical technicians in fulltime employment by EMS agencies in Nairobi County was used. The study used the sequential explanatory quantitative method design. The data for the study was obtained through questionnaires and document analysis. Data were analyzed using descriptive statistics: frequency tables and diagrams were used to present quantitative data. The questionnaire measure of internal consistency yielded a reliability of Cronbach alpha $\alpha=.82$. The study findings reveal that the EMS personnel in Nairobi County perceive that the EMS agencies' organizational strategies manifest HRO-like attributes to support reliable disaster response during MCIs. The study findings provide a mindfulness theoretical approach that EMS organizations can adapt to maintain reliable disaster response performance when dealing with the unexpected especially during response to MCIs. The study recommends that to support reliable disaster response performance especially during MCIs, EMS agencies should consider harnessing the existing organizational strategies using mindfulness strategies supported by disaster response policies in Nairobi County, Kenya.
\end{abstract}

Index terms: Mass Casualty Incidents, Emergency Medical Services, reliability, disaster response, mindfulness

\section{INTRODUCTION}

There is no country, no community, and no person immune to the impacts of disasters. Owing to a variety of human and natural factors such as technological advancements, increase in human population, and emergent human settlements in hazard-prone areas, disaster events have been occurring with increasing frequency, and with increasingly devastating consequences. Etkin (2016) defines a disaster as a serious disruption of the functioning of a community or a society, causing widespread human, material, economic or environmental losses which exceed the ability of the affected community or society to cope using its own resources. Disasters may be caused by hazards such as earthquakes, floods, wildfires, building fires, terrorism, or hazardous materials, and depending on the severity and magnitude of their impact on society, may result in significant human suffering. When these disaster events result in patient volume, injury severity, or both, at one time than locally available medical intervention resources can handle using routine procedures, they are referred to as Mass Casualty Incidents (MCIs) (Kluger, Coccolini, Catena, \& Ansaloni, 2020). This is because the number of injured patients overwhelms the handling capacity of the local emergency response system, especially the pre-hospital Emergency Medical Services (EMS) resources at the scene of the incident, and at nearby treating facilities within a short period of time (Leow et al., 2012).

When these tragic and traumatizing large-scale events occur, the public expects the local emergency services, which comprise the police (in charge of law enforcement and maintenance of public order); fire and rescue services (responsible for firefighting, technical rescue, hazardous material response); and EMS (in charge of prehospital emergency medical care), to be on hand to provide speedy and dependable assistance. EMS play a critical role in disaster response by providing timely, safe, on-scene 
evaluation, stabilization, and transportation of patients (if need be) to appropriate nearby medical facility (Government of Kenya, 2014). Mould-Millman et al. (2017) define EMS systems as "formalized prehospital care, provided by emergency care professionals who respond to medical emergencies within a well-defined jurisdiction". Additionally, an organized system of EMS personnel for reliable disaster scene management is required, in order to minimize loss of life and cater to the injured.

\section{BACKGROUND OF THE STUDY}

While Kenya's major incidents profile is dominated by fires, terrorism, infrastructural collapse, road transport incidents and disease epidemics, according to Wachira, Abdalla, and Wallis (2014), at least nine major incidents which resulted in high numbers of casualties were reported annually in the period 2009 - 2014. Examples of major disaster events that have tested the country's emergency response system include, the 2009 massive fire in Molo area where an estimated 114 people died when an overturned oil tanker burst into flames as bystanders attempted to salvage spillage for personal use. In the same year, in Nairobi CBD area, the Nakumatt Supermarket fire claimed 29 lives and scores were injured. In another disaster event on September 21, 2013, armed assailants attacked a shopping mall in the Westlands area of Nairobi, Kenya (McConnell, 2015). In this incident alone, the first civilian mass shooting incident in the country, more than 175 people were injured, 67 people were killed and many were held hostage (Wachira et al., 2014). Additionally, in Nairobi County, in 2016, residential storeyed buildings in Huruma Estate left 52 dead and scores dead, while 2019 had the disaster response structure in Nairobi County dealing with 2 major incidents - a residential multi-storeyed building collapse in Tassia Estate which left 11 dead and 29 critically injured and the Dusit D2 Hotel terror attack which left 21 dead and scores injured (Gichuki, 2019). The repetitive nature of these incidents with resultant high numbers of deaths and injuries, suggests that the emergency response system in Kenya is not adequately prepared to handle MCIs, more so in Nairobi County, where the bulk of these major incidents have taken place. Moreover, such incidents have prompted an interest towards an improvement of existing rapid response EMS system in Kenya, most notably in the period after the August 7, 1998 bombing incident of the US Embassy in Nairobi CBD area, where an estimated 240 people lost their lives and over 5000 were injured (Wachira \& Smith, 2013). It later emerged that many of the deaths in this incident were due to loss of airway, continuing haemorrhage, or respiratory failure - all preventable causes of death with appropriate emergency care (Thompson, 2013).

\section{STATEMENT OF THE PROBLEM}

Writing in 2013, Wachira and Smith noted that over 1500 people had died in Kenya, and more than one million affected by MCIs annually within the preceding 12-year period from 2001 to 2013. This observation is in agreement with Lowthian et al. (2011) remark that the incidences of natural calamities such as tsunamis, hurricanes, earthquakes, and human-made disasters such as industrial accidents, military actions and terrorist acts have been growing annually around the world. Nonetheless, beneath these observations, the common implication of interest to this study, was that when the disaster event turned to be a major incident, the number of casualties exceeded locally available resources thereby necessitating exceptional emergency intervention arrangements, and additional or extraordinary assistance to bring relief to the society. In addition, in the aftermath of such disaster events, the main goal for the EMS would be to provide urgent medical assistance by identifying and managing the critical patients, in an attempt to save lives (Lynn, 2019). This outcome however depends on their preparedness for disaster response. Compared to routine emergency response, disaster response during MCIs requires an entirely different approach to casualties' evaluation and initial medical care (Born, Briggs, Ciraulo, Frykberg, \& Hammond, 2007).

This study conceptualized the EMS professional as an important link in actualizing the EMS agency's mission and vision for provision of reliable emergency response services, and the set National Emergency Response Plan and Standard Operating Procedures and set out to examine how the EMS personnel perceived their own EMS agencies' organizational ability to execute reliable disaster response, in dynamic and unpredictable circumstances such as those presented by MCIs. The study was interested in getting the views of the EMS personnel because they are the frontline workers who respond to emergencies in the field, and who bear the burden of actualizing the mission of the EMS agencies, unlike the team at the management level, whose main duty is more at the strategic level, i.e., designing and managing systems and people, by interpreting the nation's, as well as the organizational emergency response plans and protocols (Keebler, Lazzara, \& Misasi, 2017). The aim of the study, therefore, was to examine the EMS personnel's perception of organizational attributes that influence reliable EMS response to MCIs in Nairobi County, Kenya. A similar study was carried out by Griffin (2008) to understand perceptions regarding the level of readiness to respond to large disasters and mass casualty situations. Phase 1 of the study surveyed EMS practitioners, while Phase 2 of the study - a follow-up survey, interviewed EMS managers to determine if there were significant differences in their responses.

\section{LITERATURE REVIEW}

\section{- $\quad$ Emergency response to past mass casualty incidents}

Whereas the ultimate goal of any emergency and mass casualty response is to obtain the best possible outcome for the greatest number of casualties, the efficacy of emergency response to major incidents in Kenya varies greatly across the different EMS agencies countrywide (Wachira \& Smith, 2013). While the aetiology of these past MCIs has been complex and multifactorial, each incident has posed new disaster management challenges in differing environments. According to Hugelius et al. (2020), the reality of managing any disaster situation or MCI may be complicated by the inherently unexpected and unpredictable nature of how a disaster event may unfold. Recent research in disaster management, safety science and organizational science, recognizes that small This publication is licensed under Creative Commons Attribution CC BY. 
disaster events can link together in unexpected ways to create disproportionate and disastrous events (Wachira, Abdalla, \& Wallis, 2014; TariVerdi, Miller-Hooks, \& Kirsch, 2018; Beyramijam et al., 2020). Moreover, the likelihood that secondary hazards may link together and create a chain reaction in which one problem reverberates and triggers a cascade of malfunctions through the emergency response system, greatly increases the chance that a 'minor' event may lead to a 'major' disaster in the absence of flexible response procedures, and a workforce able to function level-headedly in a volatile environment (Sullivan \& Hunter, 2009).

\section{- $\quad$ Mindful organizing as a form of operational remedy}

To deal with the likelihood of malfunction or failure of the EMS response system, safety research (Weick, Sutcliffe, \& Obstfeld, 2005; van Stralen \& Mercer, 2013; Sutcliffe, Vogus, \& Dane, 2016), as well as organizational research into establishments that work in high hazard environments, provide a framework that EMS organizations can adapt to enhance a safe and reliable response to MCIs (Venette, 2003; Sutcliffe, 2011; van Stralen \& Mercer, 2013 and Spath, 2015). In the late 1990s, to resolve mismanagement of response operations, it became clear that healthcare provision entities i.e., the ambulance services, hospital emergency departments, laboratories, and hospitals, had to proactively improve safety management in complex systems, and not just react after inefficient response, with the intention of reducing the unnecessary death and disability numbers of victims of the disaster events (Weick, Sutcliffe, \& Obstfeld, 1999). According to Oster and Braaten (2016) this move is imperative in ensuring that patients are not caught at the intersection of poorly designed processes and ineffective organizational culture. Consequently, to achieve the best outcomes, there needs to be a rapid, seamless and coordinated response action, among the EMS personnel responding on-scene; coordination among multiple responding EMS agencies, as well the integration of multiple jurisdictions into a functioning and reliable response system (O’Neill, 2005; Kapucu, 2009).

Weick and Sutcliffe (2001) have suggested mindful organizing, an approach that EMS organizations can embrace to manage, unexpected events in their work. Using this approach the response structure can handle unexpected disruptions through the adoption of organizational culture characteristics that contribute to error prevention, by having all individuals within the organization working together to achieve reliability i.e., the ability to maintain and execute error-free operations that experience fewer than anticipated accidents or events of harm, despite operating in highly complex, high-risk environments (Shrivastava, Sonpar, \& Pazzaglia, 2009). Weick and Sutcliffe (2007) refer to this team-work as collective mindfulness. Collective mindfulness is the idea that work teams can by collaborating, develop a more comprehensive picture of what is happening than that of any one individual working alone (Spath, 2015). Researchers have found five reliability principles that contribute to an organization's collective mindfulness. These are: (1) preoccupation with failure or system malfunction/system safety, (2) reluctance to simplify interpretations, (3) sensitivity to operations, (4) commitment to resilience, and (5) deference to expertise (van Stralen \& Mercer, 2013; Cantu et al. 2020; Weick \& Sutcliffe, 2007). These five processes are the fundamental tenets that support improvements in reliability, quality, and productivity in any organization (Weick \& Sutcliffe, 2001).

Their suggestion comes from a careful study of organizations that operate under very trying conditions all the time, and yet manage to have fewer than their fair share of accidents. These organizations are collectively referred to as high reliability organizations (HROs). Shrivastava et al. (2009) define a HRO as 'an organization which works in precarious environments, in which human or systemic errors can have catastrophic consequences, but which consistently seems to avoid such errors by adopting the high reliability organization's principles into its operations, to attain reliability. The term "high reliability" is used in reference to a category of organization, as opposed to as an outcome achieved. HROs which include institutions such as nuclear power generating plants, US Naval aircraft carriers, power grid dispatching centres, hospital emergency departments, and hostage negotiation teams, rarely fail even though they encounter numerous unexpected events (Roberts, 1990). These organizations have developed ways of acting and styles of leading that enable them to manage the unexpected and unpredictable better than most other kinds of organizations, by instilling the five reliability principles that contribute to collective mindfulness and fluid decision-making system (Beyea, 2005).

Furthermore, HRO literature provides evidence that when the five HRO hallmarks are at work in an organization, the organization has no choice but to function reliably because if reliability is compromised (i.e., reliability as the ability to maintain and execute error-free operations, despite operating in high-risk environments), severe harm results. The next section expounds on these $5 \mathrm{HRO}$ principles.

\section{- $\quad$ Principles of High Reliability Organizing}

As stated in the previous section, organizing for high reliability in the more effective HROs is characterised by five principles. Existing high reliability research indicates that these distinctive characteristics of HROs facilitate both problem detection and problem management (containment) (La Porte, 1996; Roberts \& Rousseau, 1989; Roberts, 1990; Weick \& Sutcliffe, 2001; Lekka, 2011; McGinnis, 2011; Sutcliffe, Paine, \& Pronovost, 2017). The first three principles i.e., preoccupation with failure/system malfunction rather than successes; reluctance to simplify interpretations; sensitivity to operations, considered to be principles of anticipation, enable problem detection, and thus help the organization be mindful of what might go wrong so that preventive actions can be taken. The last two principles, i.e., commitment to resilience, and deference to expertise, aid in problem management and thus help the organization be mindful of what to do when it is evident that improvements are needed and are hence considered to be principles of management (containment). 
1. Preoccupation with failure/system malfunction: In an organization preoccupied with failure or system malfunction, people are vigilant in observing and tracking small failures and anomalies (Spath, 2015). The goal is for individuals to be vigilant about what might go wrong because ignoring small failures leads to increased failure and possibly larger, more catastrophic events (van Stralen \& Mercer, 2013). While HROs manage to stay highly reliable by managing to remain error-free, they constantly focus on predicting and eliminating errors rather than merely reacting to them. For them, a near miss is a sign of failure, and is viewed as an opportunity to better understand what went wrong in operations. According to Spath (2015), evidence shows that a safety-conscious environment where the personnel are encouraged to speak up about mistakes and incidents without fear of retribution results in fewer mishaps and higher quality. Additionally, a near-miss or small incident, is seen as a learning moment and an opportunity to better understand what may have gone wrong in the system so as to be able to prevent a repeat in the future. Examining these happenings allows the organization to see where processes are not working right or where expectations are not being met (Weick \& Sutcliffe, 2007).

2. Reluctance to simplify: For many organizations operating in complex environments, in order to achieve success, it becomes necessary to simplify the activity in order to stay focused on a handful of key issues and key indicators (Weick \& Sutcliffe, 2001). But HROs recognize the risk of simplification and hence take deliberate steps to create a complete, and more nuanced picture of the crisis. HROs do not attempt to explain away the situation or make excuses when faced with a problem (Spath, 2015). If a simple mistake occurs, all potential causes of the problem are investigated, and all probable solutions are considered. Further, HROs understand their operating systems in the interconnectedness of their processes and do not accept simple interpretations of what might be an indicator of a systemic problem (Cantu, Gharehyakheh, Fritts, \& Tolk, 2021). The teams in HROs do not apply simplistic fixes to complex system flaws. For them, the first "good enough" solution is not acceptable. Rather, they analyse incident and event reports as well as process changes to look for unwanted system interactions so that they can be addressed before causing or exacerbating a problem. Diverse opinions and experiences are embraced to arrive at the best solution for avoiding or minimizing catastrophes. Spath (2015) stresses that the reluctance to simplify does not mean that HROs are averse to creating processes that are as simple. Rather, being reluctant to simplify means that HROs encourage workers to analyse a wide range of reasons why things go wrong, and not to presume failures are due to one simple cause that can be fixed by one simple solution. In the healthcare sector, a reluctance to simplify will also affect how patient care is provided. As such, they position themselves to see as much as possible, so that they come up with the best solution possible.

3. Sensitivity to operations: People in HROs are very aware of the complexities of their work. Everyone, from senior leaders to all staff members, individually and collectively understands the big picture of current operation. Being able to see the 'big picture' develops a comprehensive situational awareness of any undertaking, and is just as operational as it is strategic (Sutcliffe, Paine, \& Pronovost, 2017). Potential and actual anomalies are noticed while they are still manageable and can be isolated and dealt with. In HROs, the frontline employees perform the real work, therefore, the leaders keep constant and strong contact between them and their employees to make sure anomalies and problems are quickly identified and dealt with (Weick \& Sutcliffe, 2001). According to van Stralen and Mercer (2013), EMS frontline employees are positioned well to help their organizations learn by being able to suggest changes in the processes and activities. When people have well developed situational awareness, they constantly seek to understand what is happening in the moment, and what is likely to happen next, hence they can make continuous adjustments to prevent errors from accumulating and enlarging. Effective communication among work team members is essential for maintaining such situational awareness and resources are deployed to support the teams in the field.

4. Commitment to resilience: No system is perfect. A resilient organization is one that pays close attention to its ability to act regardless of what may happen (Spath, 2015). Furthermore, while most organizations try to anticipate impending problems, an HRO spends time improving the capacity of its systems and people to respond to any situation. As an unexpected situation unfolds, the demands of the situation may exceed the abilities of individuals or the organization, so it is necessary for an organization to have a back-up plan (van Stralen \& Mercer, 2013). HROs can detect, contain, and bounce back from small perturbations that occur unexpectedly in all operations, and are able to contain them at the lowest level of disruption (Cantu et al., 2021). Resilience is the ability to maintain or regain a stable work environment, regardless of what of what occurs (Van Spall, Kassam, \& Tollefson, 2015). Being resilient is all about understanding that mishaps will occur and about having options thought out ahead of time. HROs do not pretend to be error free, but their errors do not disable their operations; rather, they are contained at the lowest level possible (Bigley \& Roberts, 2001). Because there is a belief that all errors can be prevented, HROs allocate resources for activities such as simulation training and just-in-time learning, to teach people how to quickly address and contain inevitable mistakes. In an HRO, people are not caught by surprise when mistakes occur, this is because the organization is actively concerned with developing people's skills and knowledge, the teams are given options on how to react to mistakes using the best suited option, so that they can function reliably and safely in the work they do.

5. Deference to expertise: HROs recognize that the expert in the room may not be the one with the most experience or the highest rank. HROs reduce the authority gradient that interferes with communication and facilitates the migration of authority to those with the knowledge to make the best decisions (van Stralen \& Mercer, 2013). HROs cultivate this diversity not just because it helps them notice more in complex environments, but also because it helps them do more with the complexities they spot (Weick \& Sutcliffe, 2001). Rigid hierarchies such as the ICS structure, have their own vulnerabilities to error. In HROs, adherence to such rigid hierarchies is loosened especially during high-risk situations, or when the pace of operations is rapidly changing (Spath, 2015). The most experienced or senior person may not have the information needed to respond to a crisis and 
relying solely on their input would be a mistake. Looking to subject matter experts, individuals with the most expertise, despite their rank or status can help contain a potentially dangerous situation. Van Stralen and Mercer (2013) further explain that in dynamic and high-risk situations, circumstances will change and may change quickly, therefore it is important that those with intimate knowledge of the circumstances, expertise in the necessary subject matter or experience with the current situation make rapid decisions with clear communications to avoid failures.

The concept of being mindful implies having a rich awareness of discriminatory detail, and an enhanced ability to discover and correct errors that could escalate into a crisis (Wishall, 2004). Taken together, all the HRO principles are related, and are attributes of collective mindfulness required to help HROs manage the unexpected, so that they can perform reliably and error-free. Organizations concerned about reliable performance, empower their workforce to work better by: (1) Aggressively seeking to know what they do not know presently by being aware of their work process at all time; (2) They construct their reward and incentive systems to acknowledge the high price of failures as well as the rewards of reliability; and (3) They consistently communicate the general mission of the organization and encourage open communication among members.

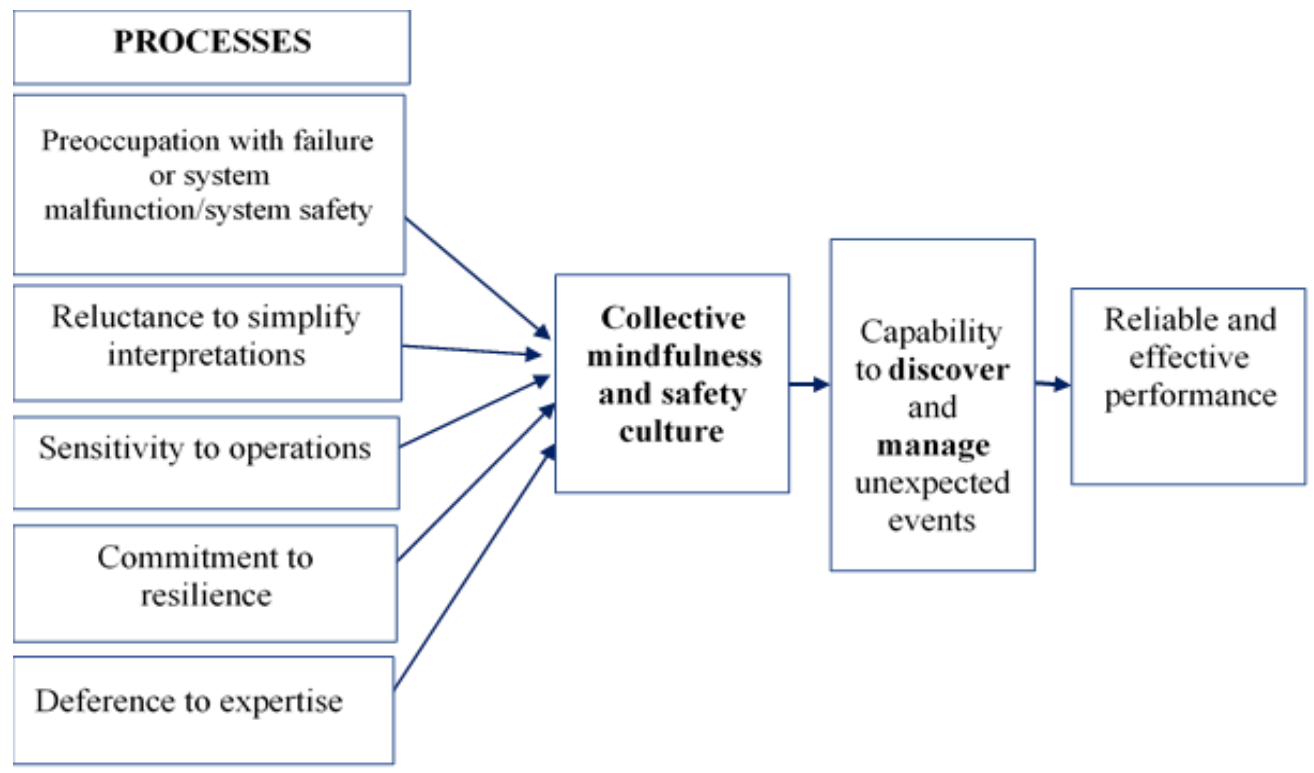

Figure 1: A mindful infrastructure for high reliability

Source: Weick, Sutcliffe, and Obstfeld (1999)

\section{- Implications of high reliability organizing for EMS personnel}

The concept of HROs is useful in the healthcare provision sector, specifically the EMS sector, in which EMS providers find themselves constantly operating in high risk, high pressure, as well as hazardous environments (van Stralen \& Mercer, 2013). Additionally, van Stralen and Mercer stress that in structure and dynamics, EMS differs little from a nuclear power production plant, space shuttle or operating room in terms of providing meticulous service. Thus, significant failure on the part of the EMS personnel and systems procedures during emergency response, can cause unintended injury, longer hospital admissions, and patient/provider injuries resulting from wrongful handling of casualties, for example dropping the patient from the stretcher during evacuation, medication errors, or even through faulty medical procedures such as esophageal intubations.

Additionally, The HRO framework was advocated for by The Institute of Medicine (IOM) in their report To Err is Human (2000) as a solution to improve medical practice, by devoting significant effort to reducing medical error, and improve patient safety. Patient safety became the focus of the public eye with the release of this report due to the statistics it presented, that 98,000 people die annually from preventable medical errors while in the care of health workers (Coles, 2014). Thus to improve patient safety, the report advocated for health care organisations to incorporate principles of high reliability organizations (HROs) into their work, in order to reduce the number of accidental injury or accidental death when handling casualties (Tamuz \& Harrison, 2006).

According to Carroll and Rudolph (2006), HROs manage work that involves interactions with hazardous environments and a high possibility of error during work performance, whereby the scale of possible consequences from errors or mistakes occasioned by an interaction of technical and human factors during operations, precludes learning through experimentation. This is especially true for the EMS system where learning from experimentation may impact negatively on patients' health. Principles adapted from HROs offer a model to reduce obstacles, and to create a safe system that improves patient outcomes (Oster \& Braaten, 2016). For EMS personnel, dealing with uncertainty, unpredictability, and unexpected consequences of destructive disasters, more so during MCIs, is a significant challenge. As it is, managing routine day-to-day emergencies - road accidents, small fires, cardiac arrests, presents a complex challenge to EMS personnel due to the need to respond under uncertain conditions, and the need to provide an immediate 
response parallel to a potential shortage of vital resources (Adini, Bodas, Nilsson, \& Peleg, 2017). These challenges are amplified during MCIs because the EMS agencies must organize for high performance in settings where the potential for error and emergent secondary-hazards is overwhelming, and medical errors brought on by the unpredictable and unexpected nature of disaster response to MCIs can lead to potentially preventable death or disability. At such a time, EMS personnel have no choice but to function reliably. According to the Centre for Patient Safety (2021), HROs anticipate that accidents or failure to perform their duty effectively may happen. Consequently, they use a specified set of principles to avoid them, by having in place organizational structures and cognitive procedures that support standardizing processes flexibly, across multiple system components, so that results are predictable, and in so doing improve reliability and high performance.

The notion of reliability in EMS i.e., the unusual capacity of the EMS team to produce collectively satisfactory outcomes of a certain minimum quality repeatedly, depends on the lack of unwanted, unanticipated, and unexplainable variance in performance, as reliability is thought to be achieved through highly standardized but flexible routines (Weick, Sutcliffe, \& Obstfeld, 1999). Although MCIs are rare, and thus provide little opportunity for real-time training and experience, the unique demands and approaches required for the initial medical care of mass casualties derives from reasonably well-orchestrated response management plans, that have evolved through experience. Lessons learned from prior disasters allow for the development of fundamental concepts that are universally applicable to emergency response to almost every catastrophic event in which there are large numbers of casualties (Born et al., 2007). Moreover, considering that each time a routine e.g., by the EMS responders is re-enacted, it unfolds in a slightly different way, correspondingly, for a system to remain reliable, it must somehow handle unforeseen and unexpected situations in ways that forestall unintended consequences. Besides, because EMS is partly a public safety service that is expected to be in a position to respond to dynamic events in hazardous conditions.

EMS also operates in an austere environments that are often without the staffing and resources thought necessary to properly stabilize and treat critically ill or injured patients (van Stralen \& Mercer, 2013). It is this adaptive and flexible activity presented by the functioning of mindful organizing that generates potential information about capability and vulnerability. Weick et al., (1999) maintain that this information will be lost unless there is a continuous process of mindful awareness of functional variations and adoption of lessons learned. It is this conceptualization of reliability, that is more grounded in adaptive human cognition and flexible action, that equates reliable outcomes with repetitive cognition and action. Reliable performance in complex systems, such as mass casualty disaster events, is complicated because reliability is a dynamic, non-event that is difficult to specify and visualize. It is dynamic because safety is preserved by human adjustments (Sutcliffe, 2011). To avert disaster, mindful organizing proactively triggers actions that forestall and limit errors and crises. Sutcliffe suggests that this would require leaders and organizational members to pay close attention to shaping the social and relational infrastructure of the organization, and to establish a set of interrelated organizing processes and practices, which jointly contribute to the system's overall culture of safety. It is important to point out that it is these processes, instituted by the five characteristics of HROs that EMS agencies must have in place, and ingrained in their personnel's practices, to enhance reliable performance during disaster response to MCIs, that this study is interested in.

By incorporating the notion of mindfulness, the EMS system increases the comprehension of the complex nature of disaster events. While the high reliability theory is not a prescription or a road map for success, rather it may be perceived as one lens through which research can understand how safe and reliable performance, under trying conditions can be pursued, thus promoting reliability in EMS response during MCIs. Effective HROs organize socially around failure rather than success, in ways that induce an ongoing state of mindfulness (Weick et al., 1999). Mindfulness, in turn, facilitates the discovery and correction of anomalies which could propagate and grow into a catastrophe. Thus, effective HROs represent complex adaptive systems that combine orderly processes of cognition with variations in routine activities that aspire for relatively error-free performance.

\section{THEORETICAL FRAMEWORK}

While there is no recognised and widely accepted theory of Emergency Management (EM), other disciplines have provided concepts, frameworks, and perspectives helpful to EM practitioners (Drabek, 2005). EM borrows theoretical contributions from the sciences such as geography, engineering, physics, and within the social sciences from sociology, psychology, business studies, when it makes use of theories such as organisational culture theories, collective behavioural theories, sustainable development theory, and rational choice theory. The use of theoretical perspectives in any profession, and in this case EM, is useful because theoretical frameworks specify actions that guide the steps that emergency managers ought to take to enhance the effectiveness of their actions (Simonovic, 2011). This study was guided by the High Reliability Theory (HRT).

\section{- High Reliability Theory}

The high reliability theory (HRT) suggests that accidents can be avoided by organizational attention to safety (Cooke \& Rohleder, 2006). The foundations of HRT were laid by Roberts (1990), LaPorte and Consolini (1991), and Weick and Sutcliffe (2007) in their studies on safety performance by organizations that operate extremely hazardous technologies such as nuclear plants and aviation industry where even the smallest of errors can lead to tragic results. The basic proposition of this theory is that while accidents may be a normal occurrence in organizations, serious ones can be prevented, or their impact minimized by implementing certain organizational practices. According to Shrivastava, Sonpar, and Pazzaglia (2009), accidents occur because organizations expose themselves to danger when they do not follow recommended safety processes. HRT therefore suggests a focus on processes This publication is licensed under Creative Commons Attribution CC BY. 
related to dynamic situations and offers insights on the period leading up to the point of accident - in this case any unintended or untoward event that disrupts the ongoing or future output of a system can be viewed as a trigger to an accident.

Additionally, HRT proposes adoption of mindfulness and reliability-enhancing approaches such as commitment to safety, a culture of continuous learning and improvement, and redundancy in safety measures (Beyea, 2005). After conducting further studies on safety performance within a high reliability theoretical framework, La Porte and other researchers from the University of California Berkeley, declared that they had discovered organisational strategies with which organizations facing complexity have achieved outstanding safety records even though they operate in very trying conditions (Roberts, 1993 as cited in Rijpma, 1997). For example, nuclear power, naval aircraft carriers, and commercial air-traffic control systems are classic examples of industries that have applied HRO principles to achieve minimal errors, despite working in highly hazardous and unpredictable conditions (Veazie, Peterson, \& Bourne, 2019). These diverse organizations have no choice but to function reliably because of the magnitude their dysfunction would cause to the public and to the environment (Weick \& Sutcliffe, 2007). If reliability is compromised, severe harm results, therefore, they termed such an organisation 'high reliability organizations'. A high reliability organization (HRO), therefore, is 'an organization that has adopted the high reliability framework into its operations to attain reliability i.e. the ability to maintain and execute error-free operations, one that experiences fewer than anticipated accidents or events of harm, despite operating in highly complex, high-risk environments' (Shrivastava et al., 2009).

This theoretical framework is appealing for this study because it helps EMS disaster response organizations and their personnel distinguish organizational strategies that promote overall reliability, by promoting a constant obsession with how the EMS strategies could fail as a result of being faced with the unexpected (Rijpma, 1997). HROs thrive on constantly being aware that they are operating in complex environments where accidents might be expected to occur frequently, yet they manage to avoid or minimize catastrophes in their operations through mindful organizing. Moreover, the theory encourages incorporation of lessons learned from the past events within collective mindfulness, constantly being aware of surroundings and intended action, as an approach to addressing and reducing errors within strategies of disaster response.

\section{- $\quad$ Research design}

\section{RESEARCH METHODOLOGY}

To accurately describe EMS organizational strategies that can foster reliable disaster response to MCIs in Nairobi County, Kenya, the researcher sought to examine how EMS personnel organize for high performance in settings where the potential for error and disaster are overwhelming, by employing a descriptive survey design. A descriptive survey design was appropriate for this study because it helps the researcher provide an accurate and systematic description of a situation or phenomenon, especially when not much is known yet about the topic or problem (Creswell \& Creswell, 2018). Moreover, a descriptive survey design observes and describes the presence, frequency or absence of characteristics of a phenomenon as it naturally occurs in order to gain additional information (Ochieng, 2015). In connection with the survey research design, Cohen et al. (2018) note that surveys are useful for gathering factual information, data on attitudes and preferences, beliefs and predictions, opinions, behaviour and experiences-both past and present. Additionally, according to Al Nizami (2011) surveys can be used to investigate the phenomenon of perception or a research that seeks categorical rather than numerical responses.

\section{- Study population}

The sampled population for the study was EMS personnel involved in disaster response operations who hold nationally recognised Emergency Medical Technician Certifications and employed in full-time status by EMS agencies in Nairobi County (Emergency Medicine Kenya Foundation, 2020). The study concentrated on this sample group as opposed to including people in management positions, because the EMTs are the ones involved in core/frontline response operations, and they are the link that actualizes the EMS agency's vision, mission, and operating procedures. An entire population may not be easy to study because of constraints involving time, access, and costs (Creswell \& Creswell, 2018).

\section{- Inclusion and Exclusion Criteria}

To obtain the most transparent results, the study participants had to meet the following criteria: (1) Be 21 years old or older, (2) be currently licensed as a certified EMS provider or as a Paramedic, and (3) be a full-time employee in an EMS agency in Nairobi County. The participants were identified by convenience sampling and were selected on availability rather than probability.

\section{- $\quad$ Sample size}

Creswell and Plano Clark (2018) posits that an entire population may not be easy to study. Therefore, a researcher must draw a representative sample. While the sample size of the population for this study was unknown due to unavailability of up-to-date record with the umbrella body that registers the EMTs - the Kenya Council of Emergency Medical Technicians, where the study population is Moreover, where the population is unknown, the sample size can be derived by computing the minimum sample size required for accuracy in estimating proportions by considering the standard normal deviation set at 95\% confidence level (1.96), percentage picking a choice or response $(50 \%=0.5)$ and the confidence interval $(0.05= \pm 5)$ (Mugenda and Mugenda, 2003).

The formula used to come up with the study population was: 


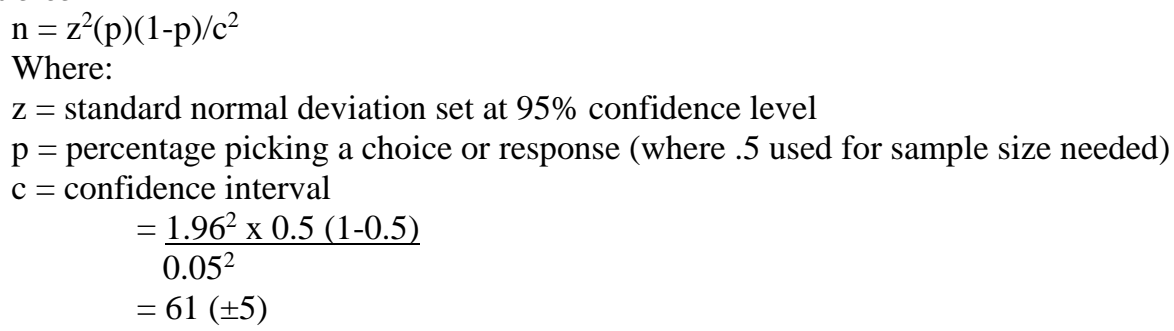

The study invited 71 potential respondents to participate in the study. Out of these 57 responses were received. 3 were incomplete. The study used 54 questionnaires (representing $77 \%$ of total study population invited to participate in the study). Mugenda and Mugenda (2003) state that while a response rate of 50\% is adequate for analysis and reporting, a response rate of $60 \%$ is good and that of $70 \%$ and above is very good.

\section{- Data collection}

The researcher made use of both primary data and secondary data. Primary data are those which are collected afresh for the first time, and are thus original in character, while secondary data are those which have already been collected by other researchers but then used by other researchers to answer different questions (Dobosh, 2017). The study questionnaire was inspired by the work of Weick and Sutcliffe (2001), who came up with an audit to assist organizations to develop an awareness of how to institutionalize mindful behaviour by spotting analogues/similarities of the five HRO processes. An adaptation of this audit was introduced by Steven Venette (2003) who used it to identify members' perception of high reliability within their organization, by examining how employees perceive risk and organizational response to risk, and termed it the HRO Perception Scale. In order for this study to examine the extent to which HRO principles are at work in EMS organizations in Nairobi County, Kenya, the first step was to administer an HRO Perception Scale Survey (Venette, 2003; Al Nizami, 2011). The study expected the results to indicate whether employees perceived their organization as an HRO. The survey questionnaire consisted of 5 questions with 7 sub-questions and used a three-point Likert-scale (not at all, to some extent, a great deal). The first three questions explored principles of anticipation (measures self-efficacy) which help the organization to be mindful of what might go wrong so that preventive actions can be taken, while the remaining two questions surveyed the principles of containment (measures organisational risk response), which help the organization be mindful of what to do when it is evident that improvements are needed (Spath, 2015).

\section{- $\quad$ Reliability of the HRO Perception Scale}

The HRO Perception Scale survey questionnaire was initially constructed by Venette (2003) and later modified by Barrett et al. (2006). Originally, the HRO Perception Scale was based on an audit based on the characteristics/principles of the HRT theory first introduced by Weick and Sutcliffe (2001), who came up with an audit to assist organizations to develop an awareness of how to institutionalize mindfulness behaviour in their personnel by spotting analogues/similarities of the five HRO processes. An adaptation of this audit was introduced by Steven Venette (2003) who used it to identify members' perception of high reliability within their organization, by examining how employees perceive risk and organizational response to risk, and termed it the HRO Perception Scale. The goal of Barrett et al. (2006) was to examine the HRO perception scale to determine whether it has reliability and construct validity, and is therefore a useful tool for organizations concerned with avoiding risk, error, near misses, and failures.

This study emulated Venette (2003) which used internal consistency technique to check on reliability of the instrument using the most common internal consistency measure which generates a coefficient value known as Cronbach's alpha ( $\alpha$ ). The recommended value of Cronbach's alpha $(\alpha)$ is 0.7 , the closer this is to 1 , the higher the internal consistency reliability (Venette, 2003; Glen, 2020). The study found the HRO survey questionnaire to have a high reliability index $(\alpha=.82, N=54)$ which Glen (2020) terms as acceptable reliability. $t$ test was used to determine the significance of this $\operatorname{HRO}$ index, $t(31)=26.324, \mathrm{p}<0.05$. Barrett et al. (2006) found it to have an acceptable reliability of $(\alpha=.83, N=84)$.

The self-efficacy subscales alpha in the Barrett et al. study was $(\alpha=.73, N=84)$, and the organizational risk response subscale alpha was $(\alpha=.86, N=84)$. This study found the self-efficacy scale to be $(\alpha=.71, N=54)$ and the organization risk response subscale alpha was $(\alpha=.84, N=54) \quad$ Both coefficients have acceptable levels of internal consistency for the overall scale and subscales (Barrett et al., 2006;Al Nizami, 2011).

\section{- $\quad$ HRO Perception Scale Validity}

Validity determines whether the research truly measures that which it was intended to measure or how truthful the research results are (Kothari, 2004; Creswell \& Plano Clark, 2018). Validity is a critical criterion and can also be thought of as utility. Kothari (2004) stresses that validity is the extent to which differences found with a measuring instrument reflect true differences among those being tested. To address the question of internal validity, the HRO Perception Scale survey, upon origination, computed twofactor analyses to test for discriminant validity. Questions on the survey were strongly fit.

\section{STUDY FINDINGS}

\section{- Study Response}

This publication is licensed under Creative Commons Attribution CC BY. 
The researcher invited 71 potential respondents to participate in the study. After executing the survey, it was paramount to review the feedback provided in the questionnaire from the angle of completeness, consistency, and homogeneity (Saris \& Gallhofer, 2014). Out of 71 respondents invited to participate in the study, 57 responses were received back. Out of this number (57), 54 questionnaires (representing 77\% response rate) were completed in full, 3 were incomplete, and thus could not be used and were discarded.

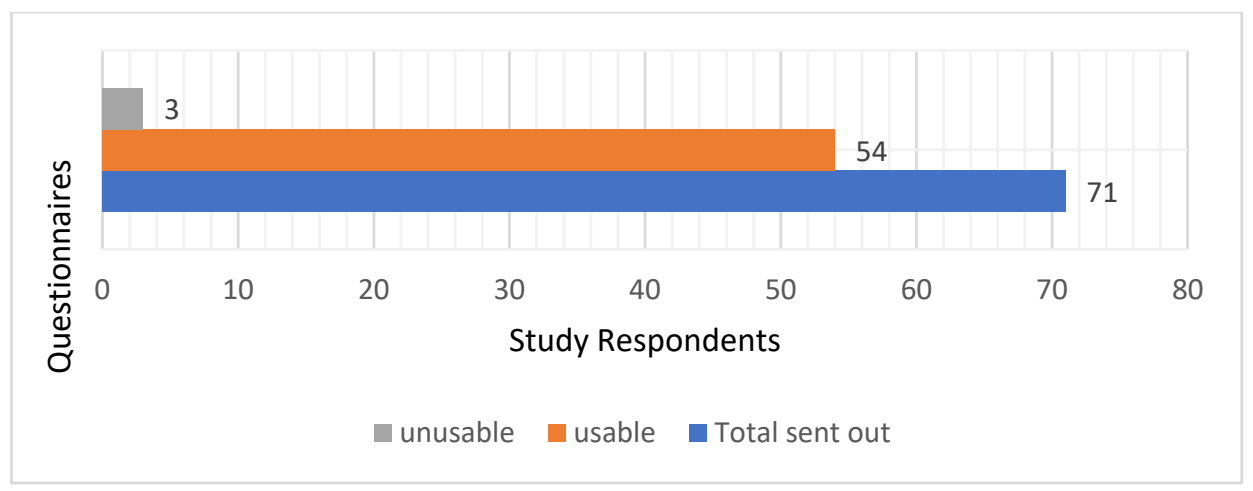

Figure 2: Study response rate on Questionnaire

Source: Field data (Researcher, 2021)

A response rate of $77 \%$ was appropriate for the study. Mugenda and Mugenda (2003) suggest that while a response rate of 50\% is adequate for analysis and reporting, a response rate of $60 \%$ is good and that of $70 \%$ and above is very good. The return rate was high because the researcher sought to sensitize the EMTs on the importance of the study to a possibility of improving their work processes for reliable outcomes.

\section{- Demographic characteristics of respondents}

This section presents demographic characteristics of respondents. Demographic information refers to characteristics of a study population and is valuable for research because it provides data important for generalizing certain characteristics of a study population. For example, a study may be able to equate certain cognitive decisions with the gender or work experience of the study respondents (Salkind, 2010). The demographic characteristics sought by this study in Section A of the questionnaire queried gender, age, academic qualifications, and years of service are presented in the following tabulation.

Table 1: Demographic characteristics of study respondents

\begin{tabular}{|l|l|c|c|}
\hline Category & Classification & Frequency & Percentage \\
\hline \multirow{5}{*}{ Gender } & Male & 38 & 70.4 \\
\hline & Female & 16 & 29.6 \\
\hline \multirow{5}{*}{ Formal EMS certification } & Below 25 & 07 & 13.0 \\
\hline & $26-35$ & 34 & 63.0 \\
\hline & $36-45$ & 12 & 22.2 \\
\hline & Above 46 & 01 & 1.9 \\
\hline & Ambulance Operator & 1 & 1.9 \\
\hline & EMT Basic & 33 & 61.1 \\
\hline & Advanced EMT & 9 & 16.7 \\
\cline { 2 - 4 } & Paramedics & 1 & 1.9 \\
\hline & Pre-hospital Registered Nurse & 1 & 1.9 \\
\hline & Other & 9 & 16.7 \\
\hline \multirow{5}{*}{ Length of Service at EMS Agency } & Less than 1 year & 9 & 16.7 \\
\cline { 2 - 4 } & $2-5$ years & 34 & 63.0 \\
\hline & $6-10$ years & 7 & 13.0 \\
\hline & $11-15$ years & 2 & 3.7 \\
\hline & $16-20$ years & 2 & 3.7 \\
\hline
\end{tabular}

\section{- $\quad$ The HRO Perception Scale survey results}

The objective of this study was to 'Examine EMS personnel's perception of high reliability attributes within their organizational strategies for disaster response during MCIs in Nairobi County, Kenya'. To address this objective, an HRO Perception Scale survey was administered online to certified EMS personnel in fulltime employment by EMS agencies operating in Nairobi County, Kenya. 
The first three questions explored principles of anticipation (measures of self-efficacy) which help the organization to be mindful of what might go wrong so that preventive actions can be taken, while the remaining two questions surveyed the principles of containment (measures organizational response to risk), which help the organization to be mindful of what to do when it is evident that improvements are needed to manage a problem. These questions, combined, determined how risk is generally understood by EMS personnel during disaster response operations, and whether the EMS organizations operated reliably during these events. The survey questionnaire consisted of 5 questions, each with 7 sub-questions which asked respondents to indicate their level of agreement with statements reflecting characteristics of HROs within their organization, on a 3-point Likert-scale - a great deal (scored 3 points), to some extent (scored 2 points), and not at all (scored 1 point).

\section{- $\quad$ HRO audit scores}

To establish the EMS personnel perceptions per HRO principle, the study made the following findings:

Table 2: Audit minimum/maximum score per HRO Principle

\begin{tabular}{cccc}
\hline HRO Principle & Minimum score & $\begin{array}{c}\text { Maximum } \\
\text { score }\end{array}$ & $\begin{array}{c}\text { Actual } \\
\text { Total }(\mathbf{n}=54)\end{array}$ \\
\hline HROP1 & 1 & 3 & 2.2619 \\
HROP2 & 1 & 3 & 2.3662 \\
HROP3 & 1 & 2 & 1.1945 \\
HROP4 & 1 & 3 & 2.4630 \\
HROP5 & 1 & 3 & 2.4106 \\
\hline
\end{tabular}

Source: Field data (Researcher, 2021)

The following section discusses the above findings per HRO principle.

The first HRO statement, HROP1, asked respondents to provide their level of agreement with the statement "Our organization is preoccupied with failure/process malfunction". The HRO mean score was 2.6219 ( $\mathrm{SD}=.6249, \mathrm{p}<.05)$. This score indicates that respondents to felt that their organizations were moderately preoccupied with system/process failure or malfunction.

The second HRO statement, HROP2, enquired if the respondents agreed with the statement “Assess your organization's reluctance to simplify" to which the respondents felt that the potential of their organization's reluctance to simplify was average with a mean score of $2.3657(\mathrm{SD}=.6219, \mathrm{p}<.05)$.

The third HRO statement, HROP3, required the respondents to agree or disagree with the statements relating to their firm's sensitivity to operations. The average score on this response was $1.6944(\mathrm{SD}=0.5732, \mathrm{p}<.05)$. The EMS personnel agreed that their firms were sensitive to disaster response operations - they had a heightened awareness of how response operations should be carried out.

The fourth question in the HRO Perception scale, HROP4, sought the EMS personnel's level of agreement with the statement, "Assess your organization's commitment to resilience". The mean score for this response was $2.4631(S D=0.6247, p<.05)$, meaning the EMTs felt that their organizations were somewhat committed to resilience - the ability to maintain or regain a stable work environment, regardless of unexpected occurrences.

The fifth question on the HRO scale sought to establish whether the EMS personnel felt that their organizations were committed to the principle of deference to expertise - the decentralization of authority in the field during disaster response, in order to encourage team-work so that those closest to the problem scene, and having the relevant skill, have 'authority' to solve problems as they emerged, while at the same time communicating their actions to other team members. The EMS personnel felt their organisations supported HROP5, the response scored a mean of $2.4106(S D=0.732, p<.05)$. This score was not as high as the researcher expected, considering that data from document analysis indicates that employees as well as the EMS organizations value working in teams and believe in the overall team-spirit in the realisation of the goals and objectives of the EMS organizations.

\section{- Discussion of overall HRO audit survey findings}

The results from the HRO Perception Scale Survey reflected the perceptions of EMTs in full-time employment in EMS agencies in Nairobi County, Kenya. When disaster events take place, the medical emergency care component of the disaster response structure plays the critical role of offering initial medical care in non-hospital/pre-hospital settings, in order to provide life-saving support, rapid diagnosis, first-aid and minor treatment to injuries, and also transfer of casualties to emergency. The functions and roles are guided by the National Standard Operating Procedures (and their organizational standard operating procedures), and the National Disaster Response Plan, that emerged from the data analysis process include: (1) Basic emergency response functions such as emergency assessment, and population monitoring and assessment, identification of population at risk (impacts on resource allocation); (2) Population protection function like taking protective actions to minimize the number of casualties; (3) Incident management role which involves mobilization and directing of resources to respond to the emergency, for example, develop flexible 
procedures to ensure the team's situational awareness activities, and also to regroup in readiness for interagency cooperation, establishing the Emergency Operation Center at the disaster scene.

It is against these functions that the results of the HRO Perception Scale survey were interrogated, to understand where the EMS agencies' organizational strategies' strengths lie, for provision of reliable disaster response during MCIs in Nairobi County, Kenya. Additionally, the underlying assumptions for research objective 1 'Examine EMS personnel's perceptions of high reliability attributes within their organizational strategies for reliable disaster response during MCIs in Nairobi County, Kenya' is that there is already a functioning EMS response structure in place, which has previously engaged in disaster response interventions during past MCIs in Nairobi County.

\section{- $\quad$ Summing EMS personnel responses for the HRO Perception Scale}

To assess the extent to which EMS personnel in Nairobi County perceived their organizations as HROs, a total score for each respondent was calculated by summing the responses to the 35 statements on the HRO Perception Scale Survey. Using a Likert scale, and audit index was developed with the scale ranging 35 - 98 with 35 being the lowest level of agreement and 98 being the highest level of agreement. The calculated mean score of the study finding $z=57.632(S D=5.72, p<.05$, at Cronbach's $\alpha=.82)$. The study findings suggest that the EMS personnel perceived their EMS agencies to moderately display HRO-like attributes. Although the EMTs indicate that their organizations display mindfulness on some HRO-attributes, on others they are quite weak. For example, findings on domains 2.5 and 2.6 which indicated that the views of the EMTs are not taken into consideration, especially input that could interrupt the operations during response. Weick and Sutcliffe (2001) suggest that in taking the HRO Perception Scale survey, EMS organizations, will be able to develop an awareness of how to institutionalize mindfulness by spotting the analogues of the five HRO attributes that help them nurture mindful processes that will support reliable disaster response performance especially during MCIs.

\section{- Study summary}

The HRO Perception Scale result was a mean score of 74.87 out of 98 indicating that the EMS personnel in Nairobi County, perceived their EMS agencies as HROs. The study findings validate the HRT premise that embracing the five HRO mindfulness processes (preoccupation with failure, reluctance to simplify, sensitivity to operations, commitment to resilience, and deference to expertise) can support an organizations objective for a highly reliable performance. Despite the study findings that the EMS organizations in Nairobi County possess characteristics of an HRO, research findings from previous studies (Mugambi, 2008; Wachira \& Martin, 2011; Thompson, 2013; Wachira \& Smith, 2013; Ochieng, 2015; Gichuki, 2019) suggest that the EMS response to past Mass Casualty Incidents in Nairobi County has been unsatisfactory.

In theory, by adapting the principles of the high reliability organizations theory into their organizational processes, EMS organizations in Nairobi County can be able to achieve reliable disaster response to MCIs by instilling mindfulness practices in all their disaster response practices and processes. By adapting the HRT, EMS organizations in Nairobi County, Kenya, may be able to enhance systemic processes that prevent errors or procedural malfunction during disaster response, by having in place mindfulness practices to manage unexpected events, especially during MCIs. Furthermore, this study finds that while the five HRO characteristics of highly reliable organizations have been demonstrated by the EMS agencies in their operational input via various organizational artefacts, their manifestation into reliable practice likely differs because the organizational structures and processes to support the valuable practice of collective mindfulness may be lacking or weak. To support EMS personnel's efforts for reliable performance to disaster response during MCIs, the EMS agencies should strive to be reliability-seeking organizations. By being 'reliability-seeking' as opposed to being 'reliable', the EMS agencies will ensure that they have in place process that transform the knowledge acquired in the moment (transient knowledge) into comprehensive situational awareness of their environment. This will ensure that disaster response to MCIs is resilient and at the same time, the structures and processes remain flexible to support continuing learning even in the middle of disaster response efforts. Over time, the embodiment of HRT principles in operational inputs will ensure that the EMS disaster response structure is prepared to handle the unexpected in ways similar to the real HROs.

\section{STUDY CONCLUSION AND RECOMMENDATION}

The study findings reveal that the EMS agencies in Nairobi County have in place the organizational processes and structures to support EMS personnel to provide reliable disaster response especially during MCIs, albeit these structures need to be harnessed using collective mindfulness strategies. Collective mindfulness encourages teamwork that allows for identification and response to signs of process/system malfunction before the onset of a full-scale disaster, to ensure reliable and failure-free functioning. In view of these finding, the study recommends that the EMS leadership should focus on understanding reliability-enhancing organizational attributes that will support their organizations to achieve and maintain operational reliability, through such activities like migratory operational expertise and flexible cognitive processes required to support collective mindfulness. These include entrenchment of reliability enhancing attributes such as those extended by the five principles of high reliability organizations that will ensure that the personnel are constantly aware and looking out for potential sources of systemic errors that may interfere with life-saving interventions during responses to disasters, encouraging and rewarding reporting of near misses and errors, and viewing every unexpected incident as an opportunity for learning, and as a means of obtaining a realistic picture of operations, thus influencing achievement and maintenance of a safety record during non-routine emergencies such as MCIs in Nairobi County, Kenya. 


\section{REFERENCES}

Adini, B., Bodas, M., Nilsson, H., \& Peleg, K. (2017). Policies for managing emergency medical services in mass casualty incidents. International Journal of Injury Care, 48(9). https://doi.org/10.1016/j.injury.2017.05.034

Al Nizami, A. K. (2011). The relevance of high reliability theory (HRT) to reliability seeking organizations (RSOs). University of Colorado, Denver .

Barrett, M. S., Novak, J. M., Venette, S. J., \& Shumate, M. (2006). Validating the High Reliability Organization Perception Scale. Communication Research Reports, 23(2). https://doi.org/10.1080/08824090600669087

Beyea, S. C. (2005). High reliability theory and highly reliable organizations. AORN Journal, 81(6), 1319-1322. https://doi.org/10.1016/S0001-2092(06)60397-9

Beyramijam, M., Khankeh, H. R., Farrokhi, M., Ebadi, A., Masoumi, G., \& Aminizadeh, M. (2020). Disaster Preparedness among Emergency Medical Service Providers: A Systematic Review Protocol. Emergency Medicine International, 2020, 1-5. https://doi.org/10.1155/2020/6102940

Bigley, G. A., \& Roberts, K. H. (2001). The Incident Command System: High-Reliability Organizing for complex and volatile task environments. Academy of Management Journal, 44(6), 1281-1299. https://doi.org/10.5465/3069401

Born, C. T., Briggs, S. M., Ciraulo, D. L., Frykberg, E. R., \& Hammond, J. S. (2007). Disasters and Mass Casualties: General principles of response and management. Journal of the American Academy of Orthopedic Surgeons, 15(7), 388-396.

Cantu, J., Gharehyakheh, A., Fritts, S., \& Tolk, J. (2021). Assessing the HRO: Tools and techniques to determine the high-reliability state of an organization. Safety Science, 134, 105082-105093. https://doi.org/10.1016/j.ssci.2020.105082

Cantu, J., Tolk, J., Fritts, S., \& Gharehyakheh, A. (2020). High Reliability Organization (HRO) systematic literature review: Discovery of culture as a foundational hallmark. Journal of Contingencies and Crisis Management, 28(4), 399-410. https://doi.org/10.1111/1468-5973.12293

Carroll, J. S., \& Rudolph, J. W. (2006). Design of high reliability organizations in health care. Quality in Health Care, 15(suppl 1). https://doi.org/10.1136/qshc.2005.015867

Centre for Patient Safety. (2021, April 5). The 5 characteristics of high reliability organizations: Quality improvement and the STAR method of decision making. Retrieved June 20, 2021, from https://www.ems1.com/safety/articles/the-5-characteristics-of-high-reliability-organizations-sn0WB7bjD4WTN6cq/

Christianson, M. K., Sutcliffe, K. M., Miller, M. A., \& Iwashyna, T. J. (2011). Becoming a high reliability organization. Critical Care, 15(6). https://doi.org/10.1186/cc10360

Cohen, L., Manion, L., \& Morrison, K. (2018). Research Methods in Education (8th ed.). New York, NY: Routledge.

Coles, E. (2014). Learning the Lessons from Major Incidents: A Short Review of the Literature. Emergency Planning College Occasional Papers New Series, (10).

Cooke, D. L., \& Rohleder, T. R. (2006). Learning from incidents: From normal accidents to high reliability. System Dynamics Review, 22(3), 213-239. https://doi.org/10.1002/sdr.338

Creswell, J., \& Plano Clark, V. (2018). Designing and conducting mixed methods research (3rd ed.). New Delhi : Sage.

Creswell, J. W., \& Creswell, D. (2018). Research Design: Qualitative, Quantitative, and Mixed Methods Approaches (5th ed.). London, UK: Sage.

Dobosh, M. A. (2017). Survey: Sampling Issues. In M. Allen (Ed.), The Sage Encyclopedia of Communication Research Methods. Thousand Oaks: Sage.

Drabek, T. E. (2005). A theory of emergency management. JEM 3(4), 49-54.

Emergency Medicine Kenya Foundation. (2020). Emergency Medical Care: The neglected disease in Kenya. Retrieved from https://www.emergencymedicinekenya.org/wp-content/uploads/2020/07/REPORT-ON-EMERGENCY-MEDICAL-CARE-IN-KENYA-PUBLIC.pdf

Etkin, D. (2016). Disaster Theory: An interdisciplinary approach to concepts and causes . Waltham, MA: Butterworth-Heinemann.

Gichuki, N. (2019). Situation analysis of the provision of prehospital Emergency Medical Services in the Nairobi County, Kenya (Jomo Kenyatta University of Agriculture and Technology). Retrieved from http://ir.jkuat.ac.ke/handle/123456789/5127

Glen, S. (2020). Cronbach's Alpha: Simple definition, use and interpretation . Retrieved September 6, 2021, from Elementary statistics for the rest of us website: https://www.statisticshowto.com/probability-and-statistics/statistics-definitions/cronbachs-alpha-spss/

Government of Kenya (Office of the President). (2014). National Emergency Response Plan and Standard Operating Procedures. Retrieved from http://www.refworld.org/pdfid/5b682fa14.pdf

Griffin, R. P. (2008). Review of Emergency Management: Principles and Practice for Local Government, Second Edition. Journal of Homeland Security and Emergency Management, 5(1). https://doi.org/10.2202/1547-7355.1457

Hugelius, K., Becker, J., \& Adolfsson, A. (2020). Five challenges when managing Mass Casualty or Disaster Situations: A Review Study. International Journal of Environmental Research and Public Health, 17(9). https://doi.org/10.3390/ijerph17093068

Kapucu, N. (2009). Interorganizational Coordination in Complex Environments of Disasters: The Evolution of Intergovernmental Disaster Response Systems. Journal of Homeland Security and Emergency Management, 6(1 (Article 47)), 1-26. https://doi.org/10.2202/1547-7355.1498

Keebler, J., Lazzara, E., \& Misasi, P. (2017). Human factors and ergonomics of prehospital emergency care . Boca Raton, FL: Routledge/CRC Press.

Kluger, Y., Coccolini, F., Catena, F., \& Ansaloni, L. (2020). WSES Handbook of Mass Casualties Incidents Management . Geneva, Switzerland: Springer.

La Porte, T. R. (1996). High Reliability Organizations: Unlikely, Demanding and At Risk. Journal of Contingencies and Crisis Management, 4(2). https://doi.org/10.1111/j.1468-5973.1996.tb00078.x

LaPorte, T., \& Consolini, P. (1991). Working in practice but not in theory: Theoretical challenges of "High-Reliability Organizations." Journal of Public Administration Research and Theory, 1(1), 19-48. https://doi.org/10.1093/oxfordjournals.jpart.a037070

Lekka, C. (2011). High reliability organisations: A review of the literature. Retrieved from https://www.hse.gov.uk/research/rrpdf/rr899.pdf

Leow, J. J., Brundage, S. I., Kushner, A. L., Kamara, T. B., Hanciles, E., Muana, A., ... Kingham, T. P. (2012). Mass casualty incident training in a resourcelimited environment. British Journal of Surgery, 99(3). https://doi.org/10.1002/bjs.7762

Lowthian, J. A., Cameron, P. A., Stoelwinder, J. U., Curtis, A., Currell, A., Cooke, M. W., \& McNeil, J. J. (2011). Increasing utilisation of emergency ambulances. Australian Health Review, 35(1), 63-69. https://doi.org/10.1071/AH09866

Lynn, M. (2019). Prehospital planning and response to sudden mass casualty incidents . In M. Lynn, H. Lieberman, L. Lynn, G. D. Pust, K. Stahl, D. D. Yeh, \& T. Zakrison (Eds.), Disasters and Mass Casualty Incidents: The nuts and bolts of preparedness and response to protracted and sudden onset emergencies. https://doi.org/10.1007/978-3-319-97361-6

McConnell, T. (2015, September 20). "Close your eyes and pretend to be dead": What really happened two years ago in the bloody attack on Nairobi's Westgate Mall. Retrieved June 29, 2021, from Foreign Policy website: http://foreignpolicy.com/2015/09/20/nairobi-kenya-westgate-mall-attack-al-shabaab

McGinnis, L. (2011). Creating High Reliability: A New Approach for Patient Safety. AORN Journal, 94(3). https://doi.org/10.1016/j.aorn.2011.05.016

Mould-Millman, N. K., Dixon, J. M., Sefa, N., Yancey, A., Hollong, B. G., Hagahmed, M., ... Wallis, L. A. (2017). The state of Emergency Medical Services (EMS) systems in Africa. Prehospital and Disaster Medicine, 32(3), 273-283. https://doi.org/10.1017/S1049023X17000061

Mugambi, B. N. (2008). Disaster Management in Kenya: A case study of the role of the military (1998 - 2008). University of Nairobi.

Mugenda, O., \& Mugenda, A. (2003). Research methods quantitative and qualitative approaches. Nairobi, Kenya: ACT Press.

O'Neill, P. A. (2005). The ABC's of disaster response. Scandinavian Journal of Surgery, 94, 259-266.

Ochieng, D. O. (2015). The influence of emergency preparedness on response strategies at general aviation airports in Kenya. Masinde Muliro University of Science and Technology, Kakamega.

Oster, C., \& Braaten, J. (2016). High Reliability Organizations: A Healthcare Handbook for Patient Safety \& Quality . Indianapolis, IN: Sigma Theta Tau International.

Rijpma, J. A. (1997). Complexity, tight-coupling and reliability: Connecting normal accidents theory and high reliability theory. Journal of Contingencies and Crisis Management, 5(1), 15-23. https://doi.org/10.1111/1468-5973.00033

Roberts, K.H., \& Rousseau, D. M. (1989). Research in nearly failure-free, high-reliability organizations: having the bubble. IEEE Transactions on Engineering Management, 36(2). https://doi.org/10.1109/17.18830 
Roberts, Karlene H. (1990). Managing High Reliability Organizations. California Management Review, 32(4). https://doi.org/10.2307/41166631

Rudolph, J. W., \& Repenning, N. P. (2002). Disaster Dynamics: Understanding the Role of Quantity in Organizational Collapse. Administrative Science Quarterly, 47(1), 1-30. https://doi.org/10.2307/3094889

Salkind, N. (Ed.). (2010). Demographics. In Encyclopedia of Research Design. https://doi.org/10.4135/9781412961288.n108

Saris, W., \& Gallhofer, I. (2014). Design, evaluation, and analysis of questionnaires for survey research. Hoboken, NJ: John Wiley \& Sons.

Shrivastava, S., Sonpar, K., \& Pazzaglia, F. (2009). Normal accident theory versus high reliability theory: A resolution and call for an open systems view of accidents. Human Relations, 62(9), 1357-1390. https://doi.org/10.1177/0018726709339117

Simonovic, S. P. (2011). Systems Approach to Management of Disasters: Methods and Applications | Wiley. Retrieved from https://www.wiley.com/enus/Systems+Approach+to+Management+of+Disasters\%3A+Methods+and+Applications-p-9781118097786

Spath, P. L. (2015). High-reliability organizations. In Handbook of Healthcare Management. https://doi.org/10.4337/9781783470167.00007

Sullivan, J. J., \& Hunter, G. (2009). Understanding the principles of high reliability in health care systems: A proposed research design . Proceedings of the Southern Association of Information Systems Conference March 12 - March 14, 2009 at Charleston, South Carolina, USA

Sutcliffe, K. M. (2011). High reliability organizations (HROs). Best Practice \& Research Clinical Anaesthesiology, 25(2). https://doi.org/10.1016/j.bpa.2011.03.001

Sutcliffe, K. M., Paine, L., \& Pronovost, P. J. (2017). Re-examining high reliability: actively organising for safety. BMJ Quality \& Safety, 26(3). https://doi.org/10.1136/bmjqs-2015-004698

Sutcliffe, K. M., Vogus, T. J., \& Dane, E. (2016). Mindfulness in Organizations: A Cross-Level Review. Annual Review of Organizational Psychology and Organizational Behavior, 3(1). https://doi.org/10.1146/annurev-orgpsych-041015-062531

Tamuz, M., \& Harrison, M. I. (2006). Improving patient safety in hospitals: Contributions of high-reliability theory and normal accident theory. Health Services Research, 41(4 II), 1654-1676. https://doi.org/10.1111/j.1475-6773.2006.00570.x

TariVerdi, M., Miller-Hooks, E., \& Kirsch, T. (2018). Strategies for Improved Hospital Response to Mass Casualty Incidents. Disaster Medicine and Public Health Preparedness, 12(6). https://doi.org/10.1017/dmp.2018.4

Thompson, M. J. (2013). A Comprehensive Review of the Emergency Medical Services System in Kenya (Emory University). Retrieved from https://etd.library.emory.edu/concern/etds/xw42n860s?locale=en

van Spall, H., Kassam, A., \& Tollefson, T. T. (2015). Near-misses are an opportunity to improve patient safety: adapting strategies of high reliability organizations to healthcare. Current Opinion in Otolaryngology \& Head and Neck Surgery, 23(4). https://doi.org/10.1097/MOO.0000000000000177

van Stralen, D., \& Mercer, T. A. (2013). EMS and High Reliability Organizing: Achieving safety and reliability in the dynamic, high-risk environment. Journal of Emergency Medical Services , June 2013, 61-63. Retrieved from https://www.researchgate.net/publication/258100801_EMS_high_reliability_organizing_Achieving_safety_reliability_in_the_dynamic_highrisk_environment

Veazie, S., Peterson, K., \& Bourne, D. (2019). Evidence Brief: Implementation of High Reliability Organization Principles. Evidence Brief: Implementation of High Reliability Organization Principles, 1-84.

Venette, S. J. (2003). Risk communication in a high reliability organization: APHIS PPQ's inclusion of risk in decision making. North Dakota State University of Agriculture and Applied Science, Fargo.

Wachira, B., Abdalla, R. O., \& Wallis, L. A. (2014). Westgate shootings: An emergency department approach to a mass-casualty incident. Prehospital and Disaster Medicine, 29(5), 538-541. https://doi.org/10.1017/S1049023X1400096X

Wachira, B., \& Martin, I. B. K. (2011). The state of emergency care in the Republic of Kenya. African Journal of Emergency Medicine, 1(4), 160-165. https://doi.org/10.1016/j.afjem.2011.10.008

Wachira, B., \& Smith, W. (2013). Major incidents in Kenya: The case for emergency services development and training. Prehospital and Disaster Medicine, 28(2), 170-173. https://doi.org/DOI: 10.1017/S1049023X13000010

Weick, K. E., \& Sutcliffe, K. M. (2001). Managing the Unexpected: Assuring high performance in an age of complexity. https://doi.org/10.1002/9781119175834

Weick, K. E., \& Sutcliffe, K. M. (2007). Managing the Unexpected: Assuring high performance in an age of complexity. Jossey-Bass.

Weick, K. E., Sutcliffe, K. M., \& Obstfeld, D. (1999). Organizing for High Reliability: Processes of collective mindfulness. In R. S. Sutton \& B. M. Staw (Eds.), Research in Organizational Behaviour (Vol. 1, pp. 81-123). London: Sage.

Weick, K. E., Sutcliffe, K. M., \& Obstfeld, D. (2005). Organizing and the Process of Sensemaking. Organization Science, 16(4). https://doi.org/10.1287/orsc. 1050.0133

Wishall, C. (2004). Mindfulness: The hallmark of a high reliability organization. Managing the Unexpected: Assuring High Performance in an Age of Complexity. Karl E. Weick and Kathleen M. Sutcliffe. Journal of Healthcare Risk Management, 24(3). https://doi.org/10.1002/jhrm.5600240307

\section{AUTHORS}

First Author - Evelyn Baru, MSc Student, Department of Emergency Management Studies, Masinde Muliro University of Science \& Technology, Kenya email: ewbaru@gmail.com

Second Author - Prof. Stanley Omuterema, Lecturer, Department of Disaster Management and Sustainable Development, Masinde Muliro University of Science \& Technology, Kenya email: somuterema @ mmust.ac.ke

Third Author - Dr Janet Nabiswa, Lecturer, Department of Educational Psychology, Kibabii University, Kenya email: jnabiswa@kibu.ac.ke

Corresponding Author - Evelyn Baru, ewbaru@gmail.com Telephone: +27670892777 